\title{
Site Progress Monitoring Using Mobile Crowd Sensing
}

\author{
${ }^{*}$ Anagha Deolikar, ${ }^{2}$ Dr. Shubhangi Neware, \\ ${ }^{1}$ M.Tech IV sem (CSE) Shri Ramdeobaba College of Engineering and Management, Nagpur \\ ${ }^{2}$ Assistant Professor (CSE) Shri Ramdeobaba College of Engineering and Management, Nagpur \\ *Email: deolikaras@rknec.edu
}

\section{Received: 09 ${ }^{\text {th }}$ July 2018, Accepted: $14^{\text {th }}$ August 2018, Published: $31^{\text {st }}$ August 2018}

\begin{abstract}
Monitoring of construction progress plays a very pivoting role in any infrastructural construction. One of the important issues in any construction activity is to know its progress status. Incorrect understanding of ongoing status can give rise to more added errors and mismanagement in the project. Normally, a project manager needs to move around the site so as to monitor the progress of the work done. In this paper, to avoid this time consuming process and in an effort to monitor the site progress in a Metro Environment, the concept of crowd sensing and image processing are considered as a whole. On the request from admin, the image of the construction site whose progress activity is to be verified is captured by the user, using Mobile Crowd Sensing (MCS). The image and location are sent to the central server. The image processing component attempts to match the captured image with the reference image. The system outputs the current bridge construction progress done. At the end, the accuracy of designed system is calculated and is proved to be factual.
\end{abstract}

Keywords: Construction Management, Progress Monitoring, Mobile Crowd Sensing, Image Processing

\section{Introduction}

The 5Ms namely- Manpower, Materials, Machinery, Money and Management are said to be the basic building elements in infrastructural construction. Each of these factors would perform their respective functions with forethought. But ensuring the work accomplished yet is another crucial task to be performed by a site manager. A timeous progress monitoring is very vital for the successful completion of any construction project. It enables a project manager to produce an action plan and accordingly make the resources available for the leftover construction activities. At a recent time, for an automated progress monitoring, mobile crowd sensing and image processing have been examined. Mobile Crowd Sensing is beneficial in recording, retrieval and transmittal of wireless data, whereas Image Processing is able to inspect the site images to elicit the progress information [1].

Conventional practice of supervision with omnipresence still is foremost dominating, the reason being lack of careful, trusty and understandable software and hardware used on the demanding situations on the sites. Previously, many projects related to infrastructural construction used equipments like digital camera or closed circuit television camera (CCTV) to record the images of construction site [2]. However it is not that easy to set up CCTV camera and also the screens to verify the progress activities. Additionally, constant presence of supervisor at various sites is required. In this paper, an effort is presented where we can use the crowd sensing and image processing combined together in order to monitor the construction operation in a Metro Environment.

Mobile crowd sensing is defined as the process of gathering suitable required data, or asking for contributions from a large group of people. Recently, with the rapid development of mobile internet and mobile social networking techniques, the scope of crowd problem solving systems using mobile devices has been more stretched out and the common internet crowd sourcing is evolving into a new standard that is Mobile Crowd Sensing. MCS promotes the large group of mobile device users to engage in crowd sensing tasks [3].

There has been viewed a strong work already performed on Mobile Crowd Sensing. The authors Minho Shin, Cory Cornelius et al. [5], in their paper propose the traffic monitoring system, which enables the mobile phone users to report their travel information to the backend server. This information is used to achieve some consumable data and gives the estimated travel time. Each phone reports its travel time or GPS reading on the road segment. Another application named SmartBike gives the distant locations of bikes, a restraint system that prevents the thefts and also gives the information about the path travelled [6]. Bin Guo [7] says that MCS uses different modalities of sensing such as numeric values (GPS coordinates), audio, images, videos. Among these all, Visual Crowd Sensing (VCS) uses cameras that are built-in smart devices. This application captures the details of interesting views or items in the real world. These are captured in the form of pictures or videos. This has achieved a huge attention because of rich information that can be provided by images and videos. S Kim, C. Robson [8] have discussed in their paper about an application named Creek Watch. It monitors the levels of water and its quality by integrating all the reports from the users by taking pictures from users. This information can then be used by water control boards to track the pollution level in water resources. 
Image processing has also attained some concrete existence in various contexts. One of them is the construction monitoring. Hongjo Kim, Kinam Kim et al. [1] in their paper have presented an interactive mobile progress monitoring system to improve the quality of progress monitoring techniques used. The concept runs in such a way that whenever a user selects any object on a construction site, the system provides the list of attributes allowing the user to match with the attributes of his interest such as location, material type and relation with other objects. This existing matching thus allows the automatic matching of other objects on the site of construction. Wu et.al [9] has developed a robust method for object recognition at the construction site. Author has used different image analytics and a 3D CAD prospect has been used which increased the accuracy of identification of object. To reflect the real progress done at the construction site, Kim et al.[10] in their paper have presented a $4 \mathrm{D}$ CAD model. The CAD model requires updating and once the model is updated it can give the progress information status. However, updating of the 4DCAD model is a process which consumes much time and is labor-demanding. Since the complete updating work requires labor and is done manually, therefore to overcome this, the authors in their paper have presented an automatic updating 4D CAD model using image processing methods. With existing 3D CAD model all the schedule information is combined automatically in batch processing modes to get 4D CAD model.

Analyzing all above-stated approaches, we have tried to mould the utilization of MCS and image processing into solely one system. The proposed research work promises to be the novel one as there has not been any work done previously by any researcher in this area. Any researcher's attention was not drawn yet to this area where bridge construction monitoring could be accomplished with the combination of MCS and image processing. Moreover, this system is more peculiar as we have encouraged the recently ongoing metro-bridge construction activity in our area.

\section{Materials and Methods:}

MCS and image processing techniques are gaining increased identification in construction monitoring. Both are being considered as the viable practice for performing the monitoring of construction sites. The concept developed here is the use of Crowd Sensing in monitoring site progress in the metro environment. The main advantage of the system is that, the supervisor does not always need to be present at the site of monitoring whereas he can simply monitor it from geographically dispersed area. The work flow of the system is as shown in figure (1).

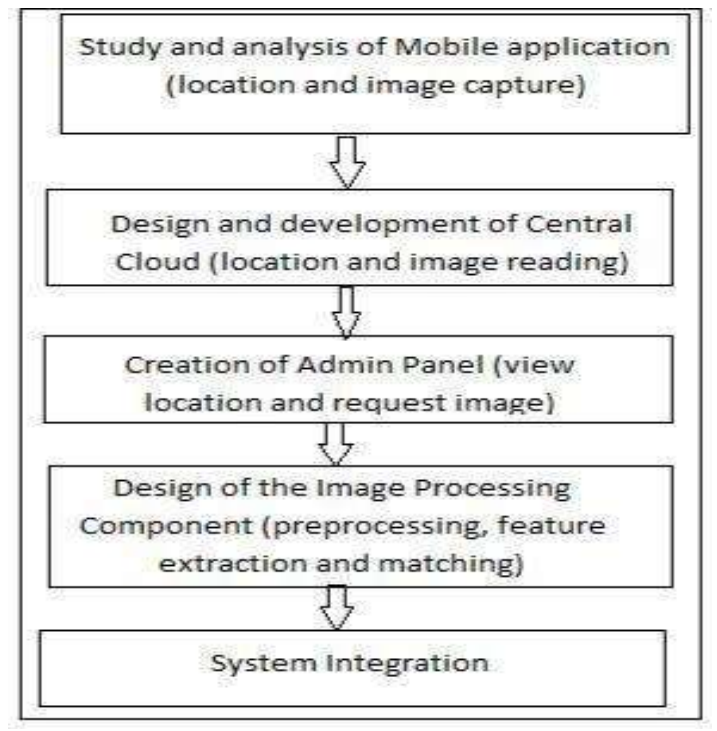

Figure1: Work Flow of the Proposed System

The block diagram (figure 2) depicts the general working idea of the proposed system.

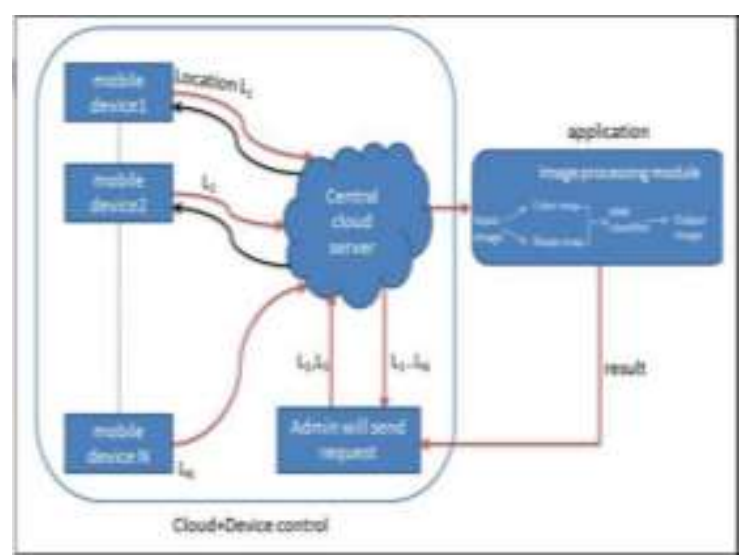

Figure 2: Block Diagram of Proposed System We have taken into account the metro construction work being done in Nagpur (Maharashtra, India). We assigned four different users at four locations to capture the images of construction sites. As requested by the admin, users took photographs of the sites. These photographs were stored on cloud and were available for the admin to check anytime. As the admin fetched the images and checked further, the image processing module presented him with the final construction progress done. The models we used were as follows:

\section{An Acquisition Process}

We developed an android application named Crowdsourced Image Processing that captured images from the different locations of interest. The sensing used in this system was participatory and the application was accessed by android users. We used four different users with four different mobile devices and the different areas were allotted to them for capturing images. Along with the images captured, the location and the timestamp were stored 
on the SD(storage device) card of the users mobile device. It also stored the latitude and longitude of location. For acquisition, User1 was allotted the area of Automotive square, Kamptee road, Nagpur (location-1) and was asked to capture images from the respective area. The images being captured were stored onto the SD card of the users mobile phone. The screenshots in figure 3(a), 3(b) and 3(c) convey the same.

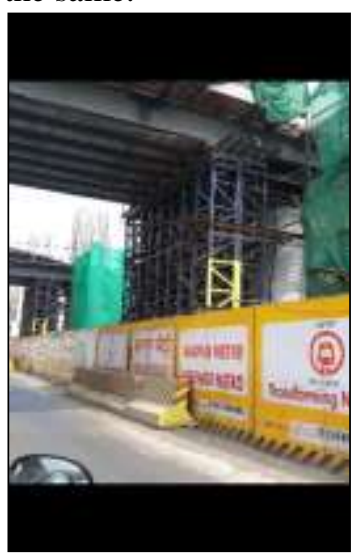

3(a)

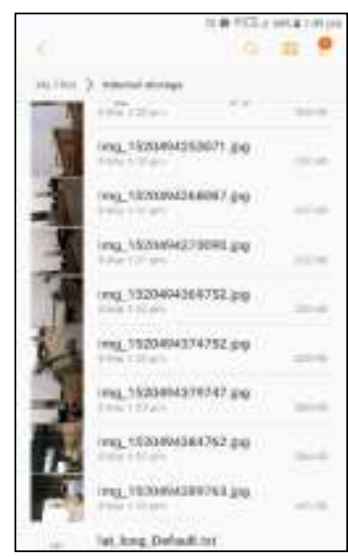

3(b)

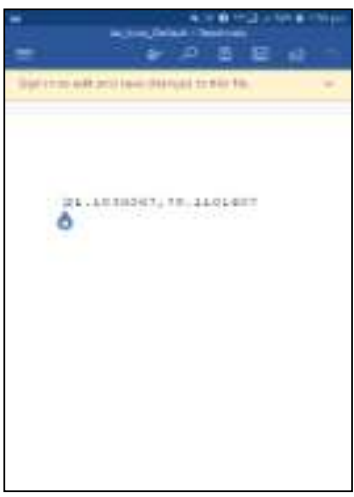

3 (c)

Figure 3: (a) Image of Metro Bridge Captured by User 1 at Location 1 (b) Captured Images Stored onto SD Card with the Name of Image Displaying the Timestamp (c) LatitudeLongitude of Area.

User 2 was allotted the area of 'Rahate Colony, Nagpur', (location-2). Similarly images, latitude, longitude and timestamp were stored on his mobile phone. The screenshots in figure(4) display the same.

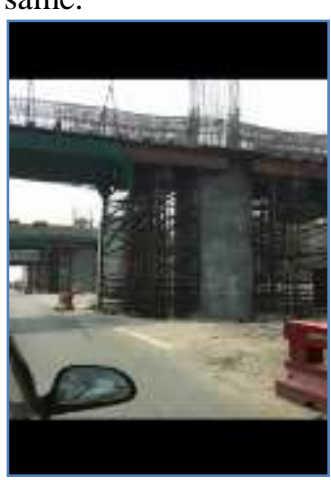

4(a)

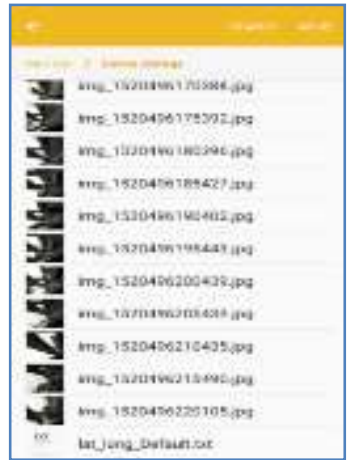

4(b)

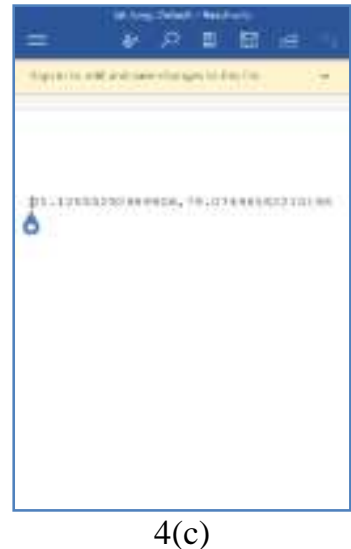

Figure 4: (a) The Image of Metro Bridge Captured by User 2 at Location 2 (b) The Stored Images on Device (c) The Latitude-Longitude of Area.

User 3 was asked to capture the images at of construction at 'Airport road, Sonegaon, Nagpur' (location-3). Figure (5) shows the screenshots of images captured, timestamp and latitude-longitude stored on device.

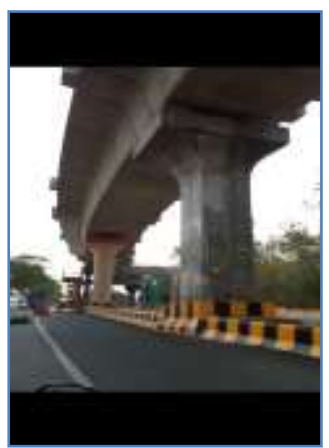

$5(a)$

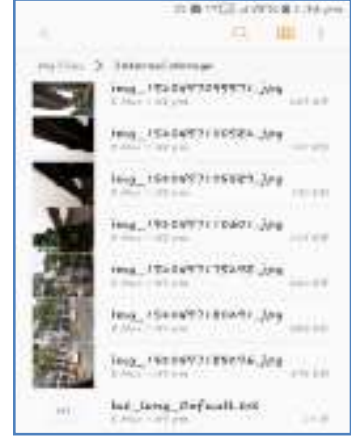

5(b)

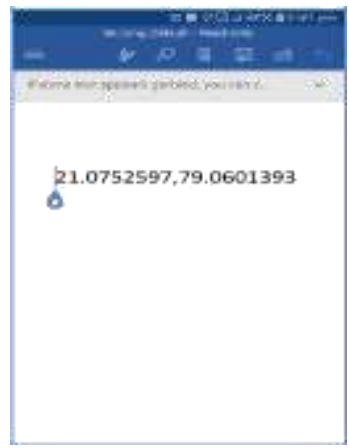

5 (c)

Figure 5: (a) The Image of Metro Bridge Construction Site Captured by User 3 at

Location 3 (b) Captured Images Stored on Mobile Device (c) Latitude-Longitude of Area respectively 
User 4 captured the construction sites ${ }^{\text {ee }}$ images at „Chhatrapati square, Nagpur (location-4). Figure

(6) shows the screenshots of user's mobile device which stored the images, latitude-longitude and timestamp on the device storage-card.

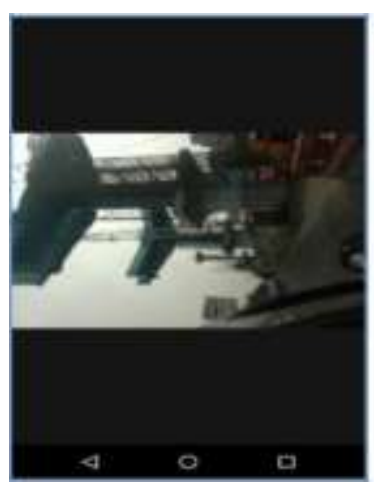

6 (a)

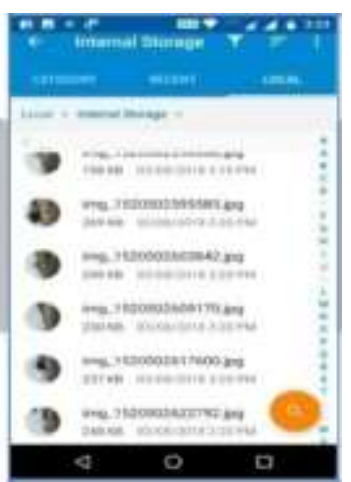

6 (b)

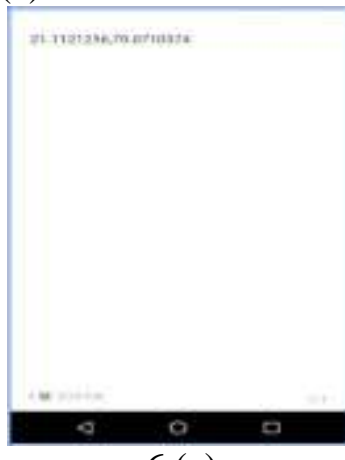

6 (c)

Figure 6: (a) Image of Metro Construction Site Captured by User 4 at location 4 (b) The Images Captured Stored onto Device of User (c) The Latitude-Longitude of the Area where Image was Captured.

\section{Central Cloud Server}

We used PHP for server-side scripting and 'WampServer' for creating a local web server where the machine itself was used as cloud for storing the data. The images captured by the users were stored onto this cloud which was directly accessible by the admin. The following screenshot (figure 7) shows the storage of data on machine as cloud.

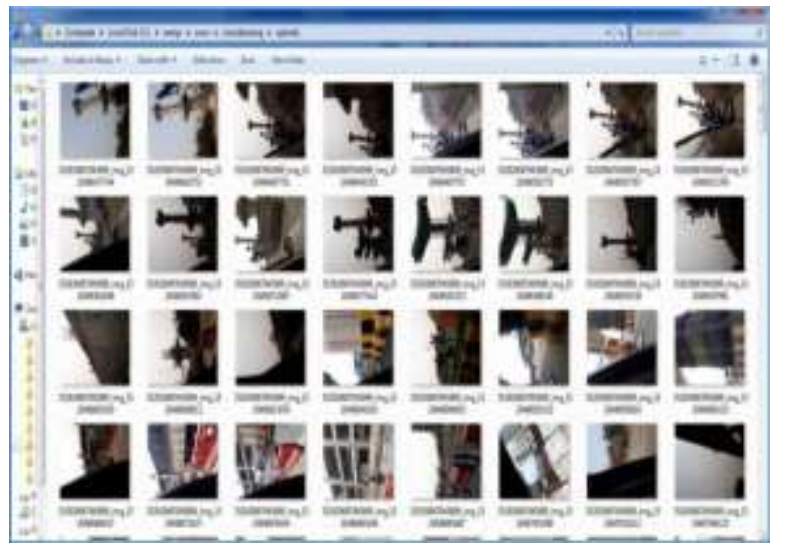

Figure 7: Screenshot showing the images captured by all the users from various locations are all stored on the admin's machine which acts as a central cloud.

\section{Image Processing Component}

From an input image, the color features and shape features were extracted. For extracting color features, we have used color-map and the shape features were extracted by using edge-map. We have used $k n n$-based ( $k$-nearest neighbour) classifier in classification process. The two sections of image processing component were „Database Traininge and „Evaluation ${ }^{\text {.e }}$ For training our database we have used 250 images of construction-sites from various locations where the construction work was going on in Nagpur. The general working of image processing in our research revolved around the certain steps of segmentation, feature extraction and classification. We have performed segmentation for identifying the objects in an image and to show the set of contours extracted from an image, we used saliency map. Saliency detection is one of the most important and significant mechanisms used for extracting the critical information effectively. In the saliency map, those features that appear to be important and visually appealing are extracted and stored whereas those which are unimportant are removed. We have used saliency map as hyper-complex representation of an image. Only those pixels are kept which catch the highest energy from an image. For detecting the saliency of an image, we have used Quaternion function [11]. The complete series of actions we conducted for getting saliency image runs as follows:

i. The input image is first resized to $128 \times 128$ for improving speed.

ii. The quaternion function is applied to obtain hyper complex representation of the image.

iii. This image is then used for entropy calculations. iv. The image is also given to Gaussian filter for smoothing.

v. The smoothened image is then combined with the highest energy image and again applied Gaussian filter 8 times for the 8 bits.

vi. The mean of the obtained image is found and compared with each of the pixels.

vii. The pixels more than mean are kept as 1 and rest are made 0 .

viii. Then the final map is obtained.

ix. This map is given to border cut for proper bordering.

$\mathrm{x}$. The final map is then overlaid on the original image to get the final saliency map image. 
For defining the color scheme for various visualizations, we have used extended color histogram. A color histogram represents the distribution of colors and the number of pixels that contains colors every fixed list of color ranges. In our experiment, the $x$-axis of color map represents color in the form of pixels, and $y$-axis represents the number of pixels of that color. The algorithm we used for color mapping is adaptive extended piecewise histogram equalization (AEPHE) [12]. Similarly, to represent corresponding comparisons of the regions by assigning different colors to different regions and analyzing shape, we used an edge map. An edge map depicts the presence of edges in an image. In our experiment, the $x$-axis of edge map represents color in the form of pixels, and $y$-axis represents the probability of that edge. Vector order statistics[13] was used for this purpose. We established a pixel collection scheme for an input image, in order to reduce the effect of broken and false edges in it and implemented vector order statistics on it. The output edge map on the basis of threshold value was produced. For classification, we have used the $k$-NN classifier. The simplest $k$-NN classification algorithm works as follows:

i. For every training example, add the example to the list of training set.

ii. Given a query instance $i_{q}$ to be classified,

a). Let $i_{1}, i_{2}, \ldots, i_{\mathrm{k}}$ denote the $k$ instances from the training set that are nearest to $i_{q}$.

b).Return the class that represents the maximum number of $k$ instances.

Figure (8) shows the saliency image, color-map and edge-map generated for one of the images captured by User1 at Automotive square, Kamptee road, Nagpur.

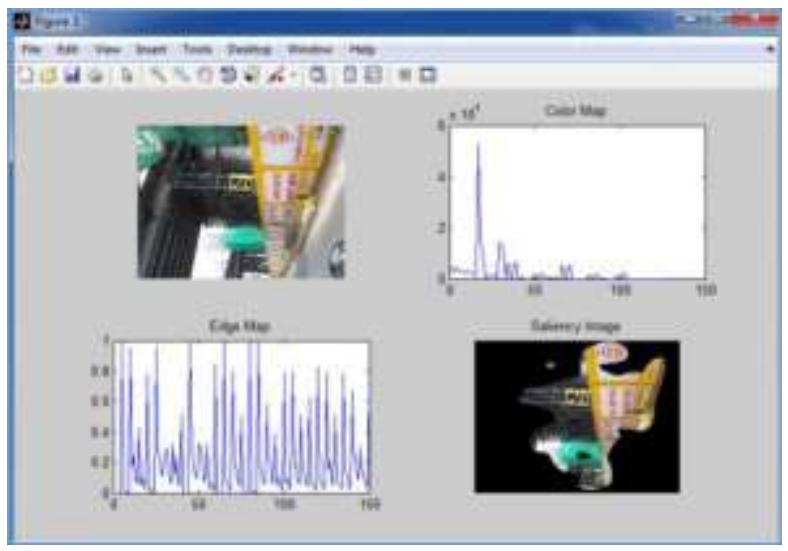

Figure 8: Color-Map, Edge-Map and Saliency Image obtained for an Input Image from User 1

\section{Admin Panel}

Our admin used his own local server and was able to check the uploads done by the users. With every image being captured by the user, the uploads also displayed its id, IMEI number of the mobile device by which it was taken, latitude_longitude of the area where the images were captured, date_time and the location of image. The figure shows the table of uploads visible to the admin. As we used four different users at four different locations, the table below shows the IMEI numbers of different mobile phones used by the users, and so the varying locations. Thus, the admin could keep an account of all the data gathered on the server, as shown in Figure(9).

\section{System Integration}

To look over the collected data and in order to check the actual progress done, the admin brought in from the server the collection of images those were captured by four different users from four distinct locations. The figure (10) represents the data fetched by admin.

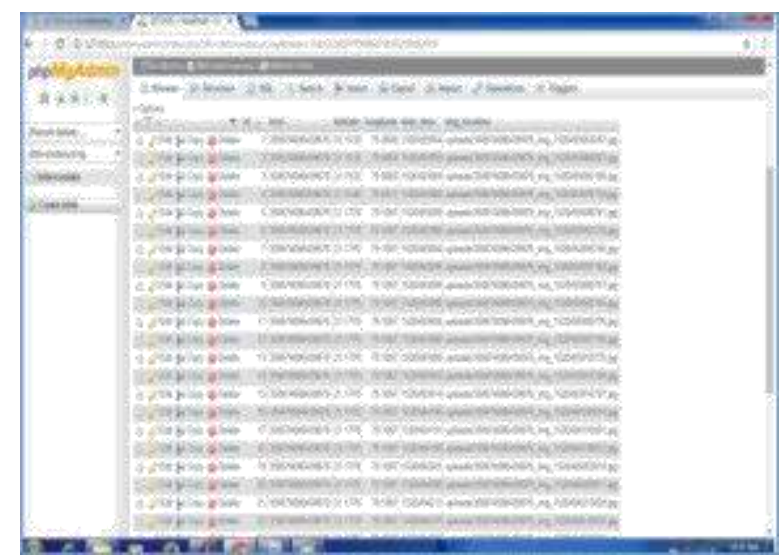

Figure 9: The Table Visible to the Admin Displaying IMEI Numbers, Timestamps and the Locations from where the Images Originated

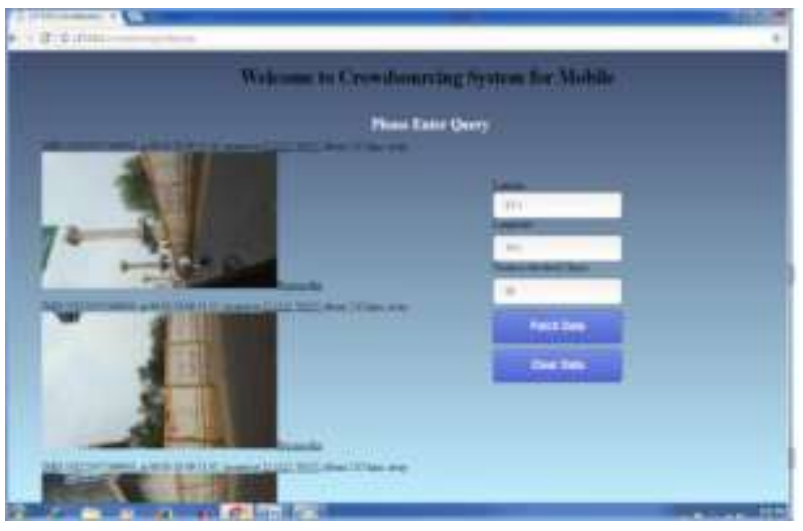

Figure 10: Collection of Images Fetched by the Admin from the Cloud. 
The admin was able to accomplish the processing of those images with a regard to verify the final progress done. He could also view the location of area where those was attained just by clicking over the ,location “. On processing, the admin was able to check the ultimate progress done. The obtained progress outputs for individual images coming from all the locations are shown below.

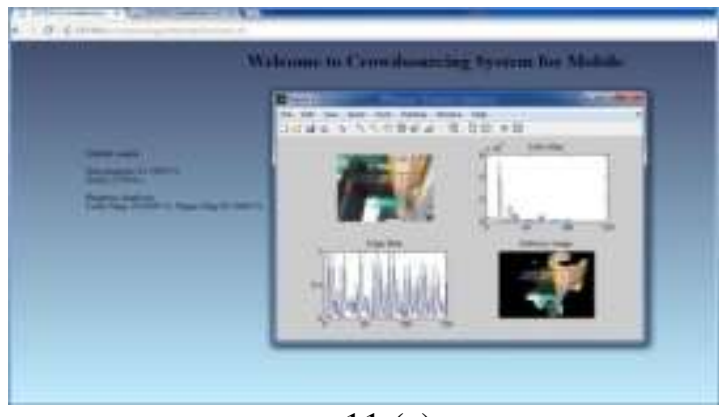

11 (a)

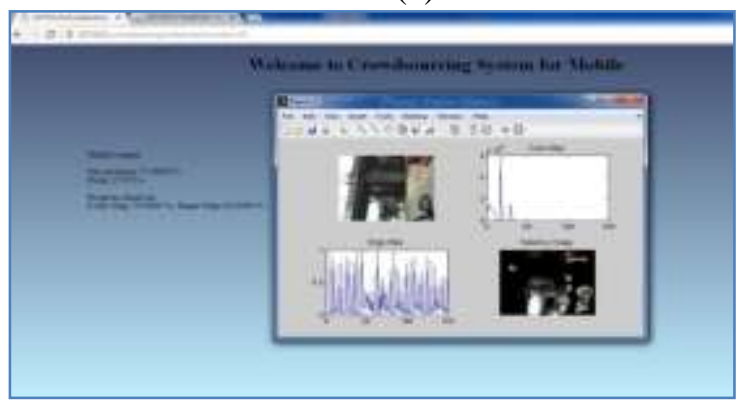

11 (b)

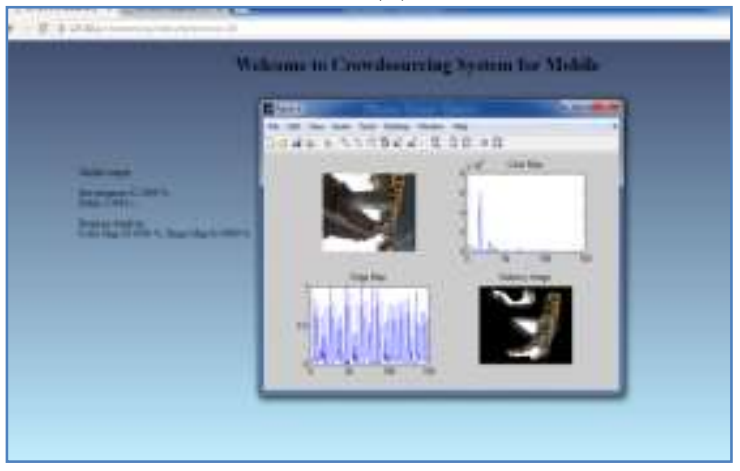

$11(\mathrm{c})$

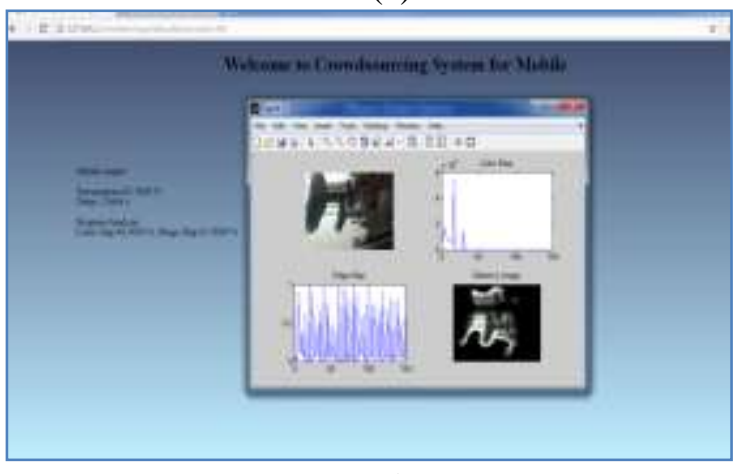

11 (d)

Figure 11: (a) Site Progress Output obtained at Location $1(42.5 \%)$ (b) Location 2 (55\%) (c) Location $3(42.5 \%)$ (d) Location 4(62.5\%).

\section{Results and Discussion}

Our complete experiment revolves around an idea of checking the actual construction progress done. For performing this experiment, we had four users (User1, User2,User3 and User4) who were allotted the distinct locations to capture the images from. For training the database, we had gathered certain images from the complete random locations where the metro-construction work was going on throughout in Nagpur area. A database with 250 images was created and trained as per the assurance of metro authority. Collective number of images sent by all the four users that simultaneously got stored on central cloud was 240 . From every location and user, we selected 25 images each, thus the total number of images evaluated was 100 . While performing data testing at location 1, 25 images were evaluated, out of which our system was able to successfully evaluate 20 images correctly.

i. Matching by the system has been shown with respect to that of the images trained of that particular area.

ii. Incorrect evaluation done by the system was because of certain distortion caused in the images due to improper way of a mobile device being held, unclear images captured or construction site getting hid as impeded by some vehicle.

\begin{tabular}{|c|c|c|c|}
\hline S No & IMEI of mobile device & timestamp & Progress output obtained (\%) \\
\hline 1 & 358674086439676 & 7:24:49 & 47.50 \\
\hline 2 & 358674086439676 & $7: 24: 50$ & 47.50 \\
\hline 3 & 358674086439676 & $7: 24: 54$ & 47.50 \\
\hline 4 & 358674086439676 & 7:24:55 & 25.00 \\
\hline 5 & 358674086439676 & $7: 24: 59$ & 32.50 \\
\hline 6 & 358674086439676 & $7: 25: 06$ & 35.00 \\
\hline 7 & 358674086439676 & 7:25:09 & 42.50 \\
\hline 8 & 358674086439676 & $7: 29: 51$ & 32.50 \\
\hline 9 & 358674086439676 & 7:29:56 & 45.00 \\
\hline 10 & 358674086439676 & 7:30:01 & 32.50 \\
\hline 11 & 358674086439676 & 7:30:06 & 42.50 \\
\hline 12 & 358674086439676 & $7: 30: 11$ & 27.50 \\
\hline 13 & 358674086439676 & $7: 30: 16$ & 40.00 \\
\hline 14 & 358674086439676 & $7: 30: 22$ & 42.50 \\
\hline 15 & 358674086439676 & $7: 30: 26$ & 32.50 \\
\hline 16 & 358674086439676 & $7: 30: 32$ & 42.50 \\
\hline 17 & 358674086439676 & $7: 30: 36$ & 42.50 \\
\hline 18 & 358674086439676 & $7: 30: 41$ & 42.50 \\
\hline 19 & 358674086439676 & $7: 30: 46$ & 30.00 \\
\hline 20 & 358674086439676 & 7:31:07 & 42.50 \\
\hline
\end{tabular}


Helix Vol. 8(5): 3904- 3911

\begin{tabular}{|l|l|r|r|}
\hline 21 & 358674086439676 & $7: 31: 11$ & 37.50 \\
\hline 22 & $\mathbf{3 5 8 6 7 4 0 8 6 4 3 9 6 7 6}$ & $\mathbf{7 : 3 2 : 5 3}$ & $\mathbf{2 5 . 0 0}$ \\
\hline 23 & $\mathbf{3 5 8 6 7 4 0 8 6 4 3 9 6 7 6}$ & $\mathbf{7 : 3 2 : 5 8}$ & $\mathbf{1 7 . 5 0}$ \\
\hline 24 & $\mathbf{3 5 8 6 7 4 0 8 6 4 3 9 6 7 6}$ & $\mathbf{7 : 3 3 : 0 3}$ & $\mathbf{2 7 . 5 0}$ \\
\hline 25 & 358674086439676 & $7: 33: 08$ & 35.00 \\
\hline
\end{tabular}

Table (1): Progress Outputs we got for Images sent by User 1 at Location 1

On evaluating the accuracy of system we got was, Number of images tested $=25$

Number of correct outputs $=20$

$\therefore$ Accuracy (in \%) $=($ Number of correct outputs /

Number of images tested) $* 100$

$$
\begin{aligned}
& =(20 / 25) * 100 \\
& =80 \%
\end{aligned}
$$

Thus, we obtained $80 \%$ accuracy at location 1 .

Similarly, on evaluation, we obtained $88 \%$ accuracy at location 2, 84\% accuracy at location 3 and $92 \%$ accuracy at location 4 .

Summarizing the accuracy matrix,

\begin{tabular}{|l|l|l|}
\hline Users & Locations & Accuracy \% obtained (A) \\
\hline User-1 & Location-1 & 80 \\
\hline User-2 & Loaction-2 & 88 \\
\hline User-3 & Location-3 & 84 \\
\hline User-4 & Location-4 & 92 \\
\hline
\end{tabular}

The average accuracy comes out to be $86 \%$, thus the overall result obtained by this system appears to be $86 \%$ accurate.

\section{Conclusion and Future Scope}

There exists various traditional site progress monitoring systems where the project manager has to walk around the site to check the progress activity or different photogrammetric methods are used on site. For an effective monitoring of a construction site, the proposed system is a promising and unique one. The system sums up a real time monitoring system where an image of a construction site from a location was sent to the server and an image processing module was competently able to match the captured image with the reference one. The obtained outcome in a percentage value happened to be more useful to interpret the current progress done at the construction site. We have made a very pertinent utilization of mobile crowd-sensing and image processing. This concept can certainly be used in monitoring any construction plans or activities to get unfailing results. With the exact information about the progress status, the project-managers can find it trouble-free to make the resources available for upcoming activities for crucial task of successful completion of project. Future scope for this research may include the use of video frames in crowd-sourcing (we have used images). Also, the construction activity may further be divided into several classes so as to make the work go on conveniently.

\section{Acknowledgement}

This work was supported in part by Nagpur Metro Rail Corporation Ltd. (NMRCL).

\section{References}

1. Kim, Hongjo, et al. "An Interactive Progress Monitoring System using Image Processing in Mobile Computing Environment." ISARC. Proceedings of the International Symposium on Automation and Robotics in Construction. Vol. 31.

Vilnius Gediminas Technical University, Department of Construction Economics \& Property, 2014.

2. Kim, Changyoon, Hyoungkwan Kim, and Yeonjong $\mathrm{Ju}$. "Bridge construction progress monitoring using image analysis." Proceedings of the 26th International Symposium on Automation and Robotics in Construction (ISARC 2009). 2009

3. Bin Guo, Xing Xie, Raghu K. Ganti, Daqing Zhang, Zhu Wang, Special Issue Proposal, Mobile Crowd sourcing (MCS),2017.

4. Ganti, Raghu K., Fan Ye, and Hui Lei. "Mobile crowdsensing: current state and future challenges." IEEE Communications Magazine 49

5. Cornelius, Cory, et al. "Anonysense: privacyaware people-centric sensing." Proceedings of the 6th international conference on Mobile systems, applications, and services. ACM, 2008.

6. Montanaro, Teodoro, et al. "SmartBike: an IoT Crowd Sensing Platform for Monitoring City Air Pollution." International Journal of Electrical and Computer Engineering (IJECE) 7.6 (2017): 36023612.

7. Guo, Bin, et al. "The Emergence of Visual Crowdsensing: Challenges and Opportunities." IEEE Communications Surveys \& Tutorials 19.4 (2017): 2526-2543.

8. S. Kim, C. Robson, T. Zimmerman, J. Pierce, and

E. M. Haber, "Creek watch: pairing usefulness and usability for successful citizen science," in

Proc. of the SIGCHI Conference on Human Factors in Computing Systems (CHI). ACM, 2011, pp. 2125-2134.

9. $\mathrm{Wu}$, Yuhong, et al. "Object recognition in construction-site images using 3D CAD-based filtering." Journal of Computing in Civil Engineering 24.1 (2009): 56-64.

10. Kim, Changyoon, Byoungil Kim, and Hyoungkwan Kim. "4D CAD model updating using image processing-based construction progress monitoring." Automation in Construction35 (2013): 44-52.

11. Zeng, Yi, and Yi Xu. "Saliency Detection 
Helix Vol. 8(5): 3904- 3911

Using Quaternion Sparse Reconstruction." Proceedings of the IEEE International Conference on Computer Vision Workshops. 2015

12. Ling, Zhigang, et al. "Adaptive extended piecewise histogram equalisation for dark image enhancement." IET Image Processing 9.11 (2015): 1012-1019.

13. Sadiq, B. O., S. M. Sani, and S. Garba. "Edge Detection: A Collection of Pixel based Approach for Colored Images." arXiv preprint arXiv: 1503.05689 (2015).

14. Sei, Yuichi, and Akihiko Ohsuga. "Differential Private Data Collection and Analysis Based on Randomized Multiple Dummies for Untrusted Mobile Crowdsensing." IEEE Transactions on Information Forensics and Security 12.4 (2017): 926-939. 